\section{SAT0546 TOTAL OSTEOPHYTE SCORE IS A BIOMARKER OF CURRENT AND PERSISTENT PAIN IN KNEE OSTEOARTHRITIS SUBJECTS}

S. Feng, M.C. Levesque. AbbVie Inc., Worcester, MA, United States

Background: X-ray and magnetic resonance imaging (MRI) demonstrated associations between osteophyte severity and current pain in knee osteoarthritis (OA) patients. Persistent pain over 5 years was also associated with x-ray osteophyte severity. A biomarker to identify knee OA subjects with persistent pain may reduce placebo response rates in clinical trials.

Objectives: To determine whether the total knee osteophyte score (TKOS), as measured by MRI Osteoarthritis Knee Score (MOAKS), is a biomarker of current and persistent pain severity in knee OA subjects.

Methods: Knee OA subjects from the Foundation for the National Institutes of Health Biomarker Consortium were longitudinally assessed over 4 years and categorized as pain and $x$-ray progressors $(n=194)$, $x$-ray-only progressors $(n=103)$, pain-only progressors $(n=103)$, and non-progressors $(n=200)$. Knee osteophyte severity was scored at baseline by MOAKS at 12 positions across the knee and summed to obtain the TKOS. TKOS was summarized as means and SDs in the 4 progression groups; each progression group TKOS was compared with the non-progression group using Wilcoxon rank sum tests (Table 1). The longitudinal Western Ontario and McMaster Universities Osteoarthritis Index (WOMAC) pain scores were plotted and tested with a linear mixed model, by high and low TKOS status, defined as above and below the TKOS median of 6 (Figure 1). Subjects with persistent pain were defined based on WOMAC pain scores at 4 out of 5 time points higher than the thresholds listed in Table 2; at each threshold, mean differences in TKOS were tested using analysis of variance among those with and without persistent pain.

Results: The distribution of baseline TKOS differed by progression group, with the highest TKOS among pain and $x$-ray progressors (Table 1). In all 4 progression groups, baseline WOMAC pain scores were higher in subjects with high $(>6)$ versus low $(\leq 6)$ baseline TKOS status $(P=0.0001,0.0383,0.0035$, and 0.0466 , respectively). The difference in WOMAC pain scores between TKOS high and low subgroups was constant over time (Figure 1; solid curves above and parallel to dashed curves with TKOS main effect always significant $[P<0.01]$ in all 4 progression groups), but the TKOS $\times$ Time interaction term in the longitudinal mixed model was not statistically significant, indicating that the pain difference between the TKOS high and low subgroups did not change over time. TKOS was highly associated with persistent pain, with TKOS higher at all thresholds (Table 2).

Table 1. Distribution Parameters of TKOS at Baseline by OA Progression Type

\begin{tabular}{lcccc}
\hline Progression Type & \multicolumn{3}{c}{ TKOS } & $P$ value) \\
\cline { 2 - 4 } & $\mathrm{n}$ & Mean & SD & \\
\hline X-ray and pain & 194 & 10.43 & 6.60 & $<0.0001$ \\
X-ray only & 103 & 8.79 & 6.56 & 0.06 \\
Pain only & 103 & 8.03 & 5.91 & 0.22 \\
None & 200 & 7.32 & 6.07 & - \\
\hline
\end{tabular}

Table 2. Group Sizes and TKOS Mean Differences for Different WOMAC Pain Thresholds for Persistent Pain

\begin{tabular}{lccc}
\hline WOMAC Pain Threshold & $\begin{array}{c}\text { Subjects With } \\
\text { Persistent Pain, } n\end{array}$ & $\begin{array}{c}\text { TKOS Difference for Subjects With } \\
\text { Persistent Pain vs Those Without }\end{array}$ & $P$ Value \\
\hline 2 & 211 & 2.5 & $<0.0001$ \\
3 & 150 & 2.4 & $<0.0001$ \\
4 & 112 & 2.4 & 0.0001 \\
6 & 52 & 3.6 & $<0.0001$ \\
8 & 21 & 2.5 & 0.06 \\
\hline
\end{tabular}

Figure 1. WOMAC pain score over 4 years stratified by baseline TKOS status and OA progression type

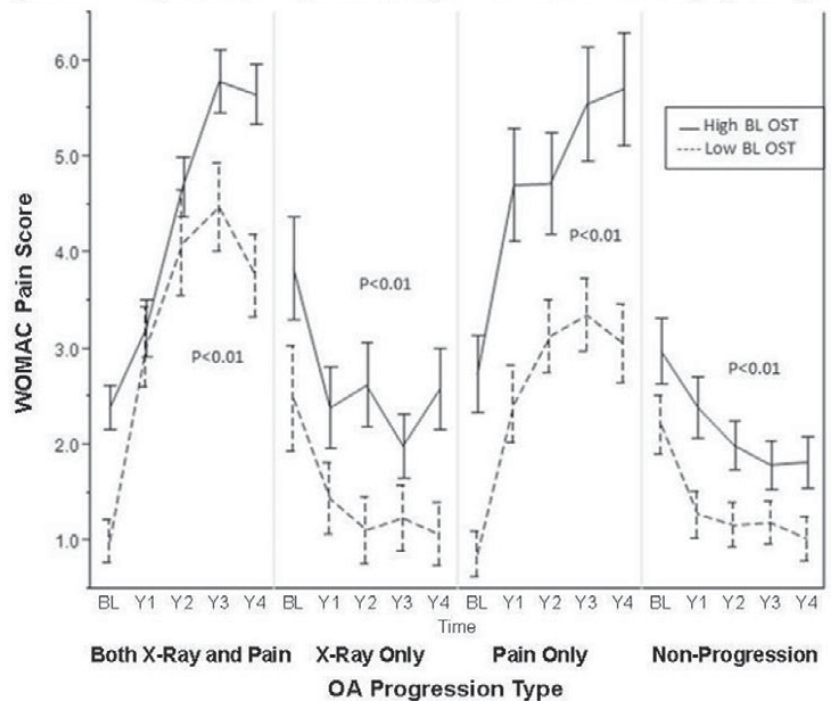

Conclusions: TKOS is a candidate biomarker for current and persistent pain in knee OA patients and may be a predictive biomarker for reduced placebo response rates in clinical trials.

Acknowledgements: AbbVie funded the analysis; participated in analysis, design and interpretation and in abstract writing, review, and approval; and funded writing support by M. Theisen of CPS.

Disclosure of Interest: S. Feng Shareholder of: AbbVie, Employee of: AbbVie, M. Levesque Shareholder of: AbbVie, Employee of: AbbVie DOI: 10.1136/annrheumdis-2017-eular.5095

\section{SAT0547 THE INFLUENCE OF OSTEOARTHRITIS ON CLINICAL, LABORATORY AND ULTRASOUND PARAMETERS OF PATIENTS WITH EARLY RHEUMATOID ARTHRITIS}

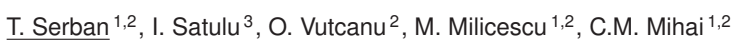

M. Bojinca ${ }^{1,2}$. 'Internal Medicine and Rheumatology, "Carol Davila" University of Medicine and Pharmacy; ${ }^{2}$ Internal Medicine and Rheumatology, "Dr. I.

Cantacuzino" Clinical Hospital, Bucharest, Romania; ${ }^{3}$ Rheumatology, Kalmar County Hospital, Kalmar, Sweden

Background: Osteoarthritis $(O A)$ and rheumatoid arthritis (RA) are not infrequent in the general population. The two pathologic entities can overlap and the presence of $O A$ can interfere with the evaluation of patients with RA

Objectives: This study aims to evaluate the possible impact of OA on the clinical, laboratory and ultrasound parameters currently evaluated in patients with early RA (ERA).

Methods: We have evaluated the data obtained from patients with ERA referred to our Early Arthritis Research Center (EARC). Only data from patients who fulfilled EULAR/ACR 2010 criteria for RA (1) and had a symptom duration of less than 12 months were analyzed. 43 patients were diagnosed with ERA in the EARC between 2012 and 2016 and were enrolled in this study. Patients were evaluated at baseline and after 12 months. All patients underwent clinical examination, laboratory tests and ultrasound (US) examination. For the US examination we have calculated the score proposed by Naredo et al. considering that this simplified US score includes the evaluation of the hand and knee. (2)

Results: There was a clear predominance of women $(62.8 \%)$. The mean age was $55.47 \pm 13.71$ years. At baseline, 21 patients $(48.8 \%)$ were diagnosed with OA. 15 patients $(34.9 \%)$ presented hand $\mathrm{OA}$ and 9 patients $(20.9 \%)$ presented knee OA. Hand OA didn't influence the values of DAS28, SDAI, patient's and physician's visual analogue scale (VAS) or ultrasound scores $(p>0.05)$. For patients with knee OA, significantly higher values for DAS28 were observed at baseline $(p=0.018)$ and were maintained significantly higher after 12 months of observation $(p=0.031)$. All the other parameters were not influenced by the presence of knee OA $(p>0.05)$. The median value and interquartile range for lab tests and for disease activity indices are shown in Table 1.

Table 1. Values for disease activity indices, laboratory tests and US scores for patients with ERA with/without OA

Lab test - Median (Interquartile range) ERA patients with OA ERA patients without OA P

\begin{tabular}{lccc} 
& $(\mathrm{n}=21)$ & $(\mathrm{n}=22)$ & \\
\hline DAS28 & $5.07(4.62-5.73)$ & $4.94(3.87-5.44)$ & 0.280 \\
SDAl & $29.02(24.95-36.59)$ & $28.87(16.18-33.97)$ & 0.644 \\
VAS patient (mm) & $72.00(55.00-81.00)$ & $62.50(50.50-72.25)$ & 0.144 \\
VAS physician (mm) & $52.00(40.00-66.50)$ & $50.00(33.75-57.00)$ & 0.456 \\
Naredo score - GS & $8.00(5.50-12.00)$ & $9.00(5.00-12.25)$ & 0.932 \\
Naredo score - PD & $4.00(0.00-6.50)$ & $3.00(0.00-4.00)$ & 0.700
\end{tabular}

Conclusions: Significantly higher values of DAS28 were observed in patients with ERA who associated knee OA, while the values of SDAl were not influenced, suggesting that SDAI may be superior to DAS28 in evaluating patients with ERA and knee OA. Not the same tendency was observed in patients with ERA and hand OA. The values of patient's VAS were not influenced by the presence of hand or knee OA suggesting that these types of OA do not influence the patients' perception of the disease activity. Moreover, the values of ultrasound scores were not influenced by the presence of $O A$.

References:

[1] Aletaha D et al. Arhritis Rheum. 2010;62:2569-2581.

[2] Naredo E et al. Clin Exp Rheumatol. 2005;23:881-884.

Disclosure of Interest: None declared

DOI: 10.1136/annrheumdis-2017-eular.4592

\section{SAT0548 PLASMA CGRP CONCENTRATIONS WERE NOT ASSOCIATED WITH PATIENT OA SYMPTOMS OR RESPONSE TO GAL- CANEZUMAB, A MONOCLONAL ANTIBODY AGAINST CGRP}

T.A. Mcnearney ${ }^{1}$, C. Smith ${ }^{2}$, R. Brown ${ }^{3}$, A. Camporeale ${ }^{4}$, M. Deeg $^{3}$,

D. Montieth ${ }^{5}$, E.C. Collins ${ }^{3}$, T.J. Schnitzer ${ }^{6}$, A.J. Kivitz ${ }^{7}$, J. Talbot ${ }^{3}$, Y. Jin $^{3}$.

${ }^{1}$ Neuroscience/Pain, Eli Lilly and Company, Indianapolis, United States; ${ }^{2}$ Eli Lilly and Company, Windlesham, United Kingdom; ${ }^{3}$ Eli Lilly and Company,

Indianapolis, United States: ${ }^{4}$ Eli Lilly and Company, Sesto Fiorentino, Italy

${ }^{5}$ Xenon Pharmaceuticals Inc, Burnaby, Canada; ${ }^{6}$ Northwestern University,

Chicago, IL; ${ }^{7}$ Altoona Center for Clinical Research, Duncansville, United States

Background: The safety and efficacy of galcanezumab, a monoclonal antibody 